"Mircea cel Batran" Naval Academy Scientific Bulletin, Volume XX - 2017 - Issue 1

The journal is indexed in: PROQUEST / DOAJ / Crossref / EBSCOhost / INDEX COPERNICUS / DRJI / OAJI I JOURNAL INDEX I I2OR / SCIENCE LIBRARY INDEX / Google Scholar / Academic Keys/ ROAD Open Access I Academic Resources / Scientific Indexing Services / SCIPIO / JIFACTOR

\title{
DATA SOLUTION FOR TURBINE SAFETY
}

\author{
Adrian POPA ${ }^{1}$ \\ Ionut-Cristian SCURTU \\ Beazit ALI ${ }^{3}$ \\ George NOVAC \\ ${ }^{1}$ Associate Professor PhD. Eng. "Mircea cel Batran" Naval Academy, Constanta, Romania, \\ adrian.popa@anmb.ro \\ ${ }^{2}$ Principal Instructor PhD. Eng."Mircea cel Batran" Naval Academy, Constanta, Romania, \\ ionut.scurtu@anmb.ro \\ ${ }^{3}$ Professor PhD. Eng "Mircea cel Batran" Naval Academy, Constanta, Romania, beazit.ali@anmb.ro \\ ${ }^{4}$ Associate Professor, PhD. Eng "Mircea cel Batran" Naval Academy, Constanta, Romania, \\ george.novac@anmb.ro
}

Abstract: Many efforts have been made in turbine safety regarding static and dynamic loads. Taking into account that turbines operate in various range of speeds and support a wide spectrum of engines, we will consider for Ansys analysis a simplified turbine model. The paper presents modal analysis for the turbine model based on Ansys software results.

Key words: turbine, modal, ansys software, meshing.

\section{Introduction}

A turbine is designed to increase air intake causing the engine to use more fuel and produce more power output.

Turbines are high precision machine parts and they must be used accordingly. Even if high quality turbines are used, their expected performance cannot be achieved if they are not handled in safety conditions. Vibration can cause faults in any equipment we will investigate via Ansys software the turbine own frequencies. Presented data will allow correctly application to met maximum efficiency, reliability, durability and performance.

\section{Material and geometry definition}

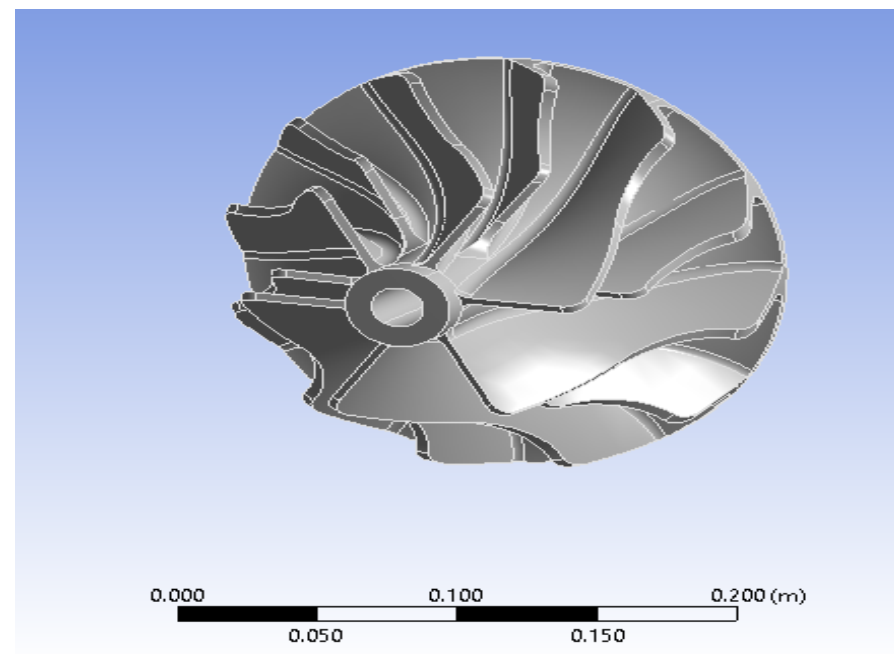

\begin{tabular}{|c|c|}
\hline First Saved & $\begin{array}{c}\text { Saturday, October 15, } \\
2016\end{array}$ \\
\hline Last Saved & $\begin{array}{l}\text { Saturday, October 15, } \\
2016\end{array}$ \\
\hline Product Version & 16.2 Release \\
\hline $\begin{array}{r}\text { Save Project Before } \\
\text { Solution }\end{array}$ & No \\
\hline $\begin{array}{r}\text { Save Project After } \\
\text { Solution }\end{array}$ & No \\
\hline
\end{tabular}

1. UNITS

Figure 1. Turbine geometry 3D CAD model

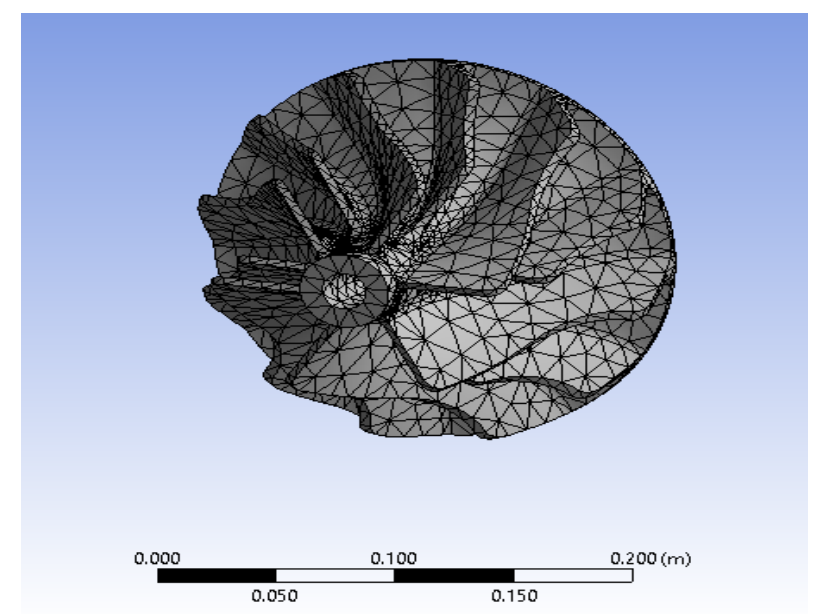

Figure 2. Turbine mesh on 3D CAD model

\begin{tabular}{|r|c|}
\multicolumn{2}{|c|}{ TABLE 1 } \\
\hline Unit System & $\begin{array}{c}\text { Metric }(\mathrm{m}, \mathrm{kg}, \mathrm{N}, \mathrm{s}, \mathrm{V}, \mathrm{A}) \\
\text { Degrees rad/s Celsius }\end{array}$ \\
\hline Angle & Degrees \\
\hline
\end{tabular}


"Mircea cel Batran" Naval Academy Scientific Bulletin, Volume XX - 2017 - Issue 1

The journal is indexed in: PROQUEST / DOAJ / Crossref / EBSCOhost / INDEX COPERNICUS / DRJI / OAJI I JOURNAL INDEX I I2OR / SCIENCE LIBRARY INDEX / Google Scholar / Academic Keys/ ROAD Open Access I Academic Resources / Scientific Indexing Services / SCIPIO / JIFACTOR

\begin{tabular}{|r|c|}
\hline $\begin{array}{r}\text { Rotational } \\
\text { Velocity }\end{array}$ & $\mathrm{rad} / \mathrm{s}$ \\
\hline Temperature & Celsius \\
\hline
\end{tabular}

\section{GEOMETRY}

TABLE 2

Model (A4) > Geometry

\begin{tabular}{|c|c|}
\hline Object Name & Geometry \\
\hline State & Fully Defined \\
\hline \multicolumn{2}{|l|}{ Definition } \\
\hline \multicolumn{2}{|l|}{ Source } \\
\hline Type & DesignModeler \\
\hline Length Unit & Meters \\
\hline Element Control & $\begin{array}{l}\text { Program } \\
\text { Controlled }\end{array}$ \\
\hline Display Style & Body Color \\
\hline \multicolumn{2}{|c|}{ Bounding Box } \\
\hline Length $X$ & $0.20879 \mathrm{~m}$ \\
\hline Length $\mathrm{Y}$ & $0.1143 \mathrm{~m}$ \\
\hline Length Z & $0.20879 \mathrm{~m}$ \\
\hline \multicolumn{2}{|l|}{ Properties } \\
\hline Volume & $8.8665 \mathrm{e}-004 \mathrm{~m}^{3}$ \\
\hline Mass & $2.456 \mathrm{~kg}$ \\
\hline Scale Factor Value & 1. \\
\hline \multicolumn{2}{|l|}{ Statistics } \\
\hline Bodies & 1 \\
\hline Active Bodies & 1 \\
\hline Nodes & 27582 \\
\hline Elements & 15881 \\
\hline Mesh Metric & None \\
\hline \multicolumn{2}{|c|}{ Basic Geometry Options } \\
\hline Parameters & Yes \\
\hline Parameter Key & DS \\
\hline Attributes & No \\
\hline Named Selections & No \\
\hline Material Properties & No \\
\hline \multicolumn{2}{|c|}{ Advanced Geometry Options } \\
\hline Use Associativity & Yes \\
\hline Coordinate Systems & No \\
\hline $\begin{array}{r}\text { Reader Mode Saves Updated } \\
\text { File }\end{array}$ & No \\
\hline Use Instances & Yes \\
\hline Smart CAD Update & No \\
\hline Compare Parts On Update & No \\
\hline Attach File Via Temp File & Yes \\
\hline Temporary Directory & \\
\hline Analysis Type & $3-D$ \\
\hline Decompose Disjoint Geometry & Yes \\
\hline $\begin{array}{r}\text { Enclosure and Symmetry } \\
\text { Processing }\end{array}$ & Yes \\
\hline
\end{tabular}

TABLE 3

Model (A4) > Geometry > Parts

\begin{tabular}{r|c} 
Object Name & turbo-rotor \\
State & Meshed
\end{tabular}

Graphics Properties

Visible

Transparency Yes

\begin{tabular}{|r|c|}
\hline Visible & Yes \\
\hline Transparency & 1 \\
\hline Definition \\
\hline Suppressed & No \\
\hline Stiffness Behavior & Flexible \\
\hline Coordinate System & $\begin{array}{c}\text { Default Coordinate } \\
\text { System }\end{array}$ \\
\hline
\end{tabular}

\begin{tabular}{|r|c|}
\hline $\begin{array}{r}\text { Reference } \\
\text { Temperature }\end{array}$ & By Environment \\
\hline Behavior & None \\
\hline Material \\
\hline Assignment & Aluminum Alloy \\
\hline Nonlinear Effects & Yes \\
\hline Thermal Strain Effects & Yes \\
\hline
\end{tabular}

Bounding Box

\begin{tabular}{|r|c|}
\hline Length $\mathrm{X}$ & $0.20879 \mathrm{~m}$ \\
\hline Length $\mathrm{Y}$ & $0.1143 \mathrm{~m}$ \\
\hline Length $\mathrm{Z}$ & $0.20879 \mathrm{~m}$ \\
\hline
\end{tabular}

\section{Properties}

\begin{tabular}{|r|c|}
\hline Volume & $8.8665 \mathrm{e}-004 \mathrm{~m}^{3}$ \\
\hline Mass & $2.456 \mathrm{~kg}$ \\
\hline Centroid X & $-1.3141 \mathrm{e}-006 \mathrm{~m}$ \\
\hline Centroid Y & $2.8241 \mathrm{e}-002 \mathrm{~m}$ \\
\hline Centroid Z & $-5.3549 \mathrm{e}-008 \mathrm{~m}$ \\
\hline Moment of Inertia Ip1 & $5.6371 \mathrm{e}-003 \mathrm{~kg} \cdot \mathrm{m}^{2}$ \\
\hline Moment of Inertia Ip2 & $7.6846 \mathrm{e}-003 \mathrm{~kg} \cdot \mathrm{m}^{2}$ \\
\hline Moment of Inertia Ip3 & $5.6359 \mathrm{e}-003 \mathrm{~kg} \cdot \mathrm{m}^{2}$ \\
\hline Statistics \\
\hline Nodes & 27582 \\
\hline Elements & 15881 \\
\hline Mesh Metric & None
\end{tabular}

\section{COORDINATE SYSTEMS}

\section{TABLE 4}

Model (A4) > Coordinate Systems > Coordinate System

Object Name Global Coordinate System State Fully Defined

\section{Definition}

Type

Coordinate System ID Cartesian 0.

\begin{tabular}{ll|}
\multicolumn{2}{|c|}{ Origin } \\
Origin $\mathrm{X}$ & $0 . \mathrm{m}$ \\
\hline Origin $\mathrm{Y}$ & $0 . \mathrm{m}$ \\
Origin $\mathrm{Z}$ & $0 . \mathrm{m}$ \\
Directional Vectors
\end{tabular}


"Mircea cel Batran" Naval Academy Scientific Bulletin, Volume XX - 2017 - Issue 1

The journal is indexed in: PROQUEST / DOAJ / Crossref / EBSCOhost / INDEX COPERNICUS / DRJI / OAJI I JOURNAL INDEX / I2OR / SCIENCE LIBRARY INDEX / Google Scholar / Academic Keys/ ROAD Open Access I Academic Resources / Scientific Indexing Services / SCIPIO / JIFACTOR

\begin{tabular}{|r|c|}
\hline X Axis Data & {$[1.0 .0]$.} \\
\hline Y Axis Data & {$[0.1 .0]$.} \\
\hline Z Axis Data & {$[0.0 .1]$.} \\
\hline
\end{tabular}

4. MESH

TABLE 5

Model (A4) > Mesh

\begin{tabular}{|c|c|}
\hline Object Name & Mesh \\
\hline State & Solved \\
\hline \multicolumn{2}{|l|}{ Display } \\
\hline Display Style & Body Color \\
\hline \multicolumn{2}{|l|}{ Defaults } \\
\hline Physics Preference & Mechanical \\
\hline Relevance & 100 \\
\hline Shape Checking & $\begin{array}{c}\text { Standard } \\
\text { Mechanical }\end{array}$ \\
\hline Element Midside Nodes & Program Controlled \\
\hline \multicolumn{2}{|c|}{ Sizing } \\
\hline Size Function & Adaptive \\
\hline Relevance Center & Coarse \\
\hline Element Size & Default \\
\hline Initial Size Seed & Active Assembly \\
\hline Smoothing & Medium \\
\hline Transition & Fast \\
\hline Span Angle Center & Coarse \\
\hline $\begin{array}{r}\text { Automatic Mesh Based } \\
\text { Defeaturing }\end{array}$ & On \\
\hline Defeaturing Tolerance & Default \\
\hline Minimum Edge Length & 2.8973e-005 m \\
\hline \multicolumn{2}{|l|}{ Inflation } \\
\hline Use Automatic Inflation & None \\
\hline Inflation Option & Smooth Transition \\
\hline Transition Ratio & 0.272 \\
\hline Maximum Layers & 5 \\
\hline Growth Rate & 1.2 \\
\hline Inflation Algorithm & Pre \\
\hline View Advanced Options & No \\
\hline \multicolumn{2}{|l|}{ Advanced } \\
\hline $\begin{array}{r}\text { Number of CPUs for Parallel } \\
\text { Part Meshing }\end{array}$ & Program Controlled \\
\hline Straight Sided Elements & No \\
\hline Number of Retries & Default (4) \\
\hline Extra Retries For Assembly & Yes \\
\hline Rigid Body Behavior & $\begin{array}{l}\text { Dimensionally } \\
\text { Reduced }\end{array}$ \\
\hline Mesh Morphing & Disabled \\
\hline Triangle Surface Mesher & Program Controlled \\
\hline Topology Checking & No \\
\hline Pinch Tolerance & Please Define \\
\hline Generate Pinch on Refresh & No \\
\hline \multicolumn{2}{|l|}{ Statistics } \\
\hline
\end{tabular}

\begin{tabular}{|r|c|}
\hline Nodes & 27582 \\
\hline Elements & 15881 \\
\hline Mesh Metric & None \\
\hline
\end{tabular}

5. MODAL (A5)

TABLE 6

Model (A4) > Analysis

\begin{tabular}{|c|c|}
\hline Object Name & Modal (A5) \\
\hline State & License Full \\
\hline \multicolumn{2}{|c|}{ Definition } \\
\hline Physics Type & Structural \\
\hline Analysis Type & Modal \\
\hline Solver Target & Mechanical APDL \\
\hline \multicolumn{2}{|c|}{ Options } \\
\hline Environment Temperature & 22. ${ }^{\circ} \mathrm{C}$ \\
\hline Generate Input Only & No \\
\hline
\end{tabular}

\section{Solution (A6)}

TABLE 9

Model (A4) $>$ Modal (A5) $>$ Solution

\begin{tabular}{|r|c|}
\hline Object Name & Solution (A6) \\
\hline State & Solved \\
\hline \multicolumn{2}{|c|}{ Adaptive Mesh Refinement } \\
\hline Max Refinement Loops & 1. \\
\hline Refinement Depth & 2. \\
\hline \multicolumn{2}{|c|}{ Information } \\
\hline Status & Done \\
\hline MAPDL Elapsed Time \\
\hline MAPDL Memory Used \\
\hline \multicolumn{2}{|c|}{ MAPDL Result File Size } \\
\hline Calculate Beam Section Results \\
\hline
\end{tabular}

The following bar chart indicates the frequency at each calculated mode. 
"Mircea cel Batran" Naval Academy Scientific Bulletin, Volume XX - 2017 - Issue 1

The journal is indexed in: PROQUEST / DOAJ / Crossref / EBSCOhost / INDEX COPERNICUS / DRJI / OAJI I JOURNAL INDEX I I2OR / SCIENCE LIBRARY INDEX / Google Scholar / Academic Keys/ ROAD Open Access I Academic Resources / Scientific Indexing Services / SCIPIO / JIFACTOR

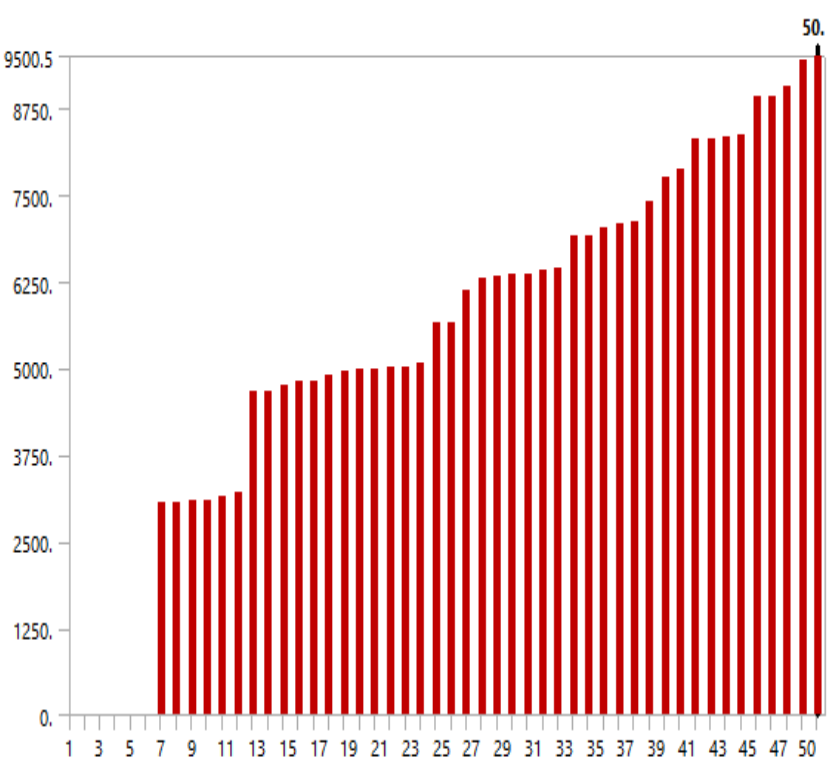

Figure 3. Modal solution

TABLE 10

Model (A4) $>$ Modal (A5) $>$ Solution (A6)

\begin{tabular}{|c|c|}
\hline Mode & Frequency [Hz] \\
\hline 1. & \multirow[t]{3}{*}{0} \\
\hline 2. & \\
\hline 3. & \\
\hline 4. & $1.0199 \mathrm{e}-003$ \\
\hline 5. & $1.6096 \mathrm{e}-003$ \\
\hline 6. & $1.7761 \mathrm{e}-003$ \\
\hline 7. & 3066.2 \\
\hline 8. & 3068.8 \\
\hline 9. & 3099.9 \\
\hline 10. & 3102.4 \\
\hline 11. & 3152.3 \\
\hline 12. & 3208.4 \\
\hline 13. & 4650.6 \\
\hline 14. & 4654. \\
\hline 15. & 4738.5 \\
\hline 16. & 4816.6 \\
\hline 17. & 4821.2 \\
\hline 18. & 4892.3 \\
\hline 19. & 4961.9 \\
\hline 20. & 4970.4 \\
\hline 21. & 4990.8 \\
\hline 22. & 5004.1 \\
\hline 23. & 5018.3 \\
\hline 24. & 5068.9 \\
\hline 25. & 5640. \\
\hline 26. & 5646.4 \\
\hline 27. & 6114.6 \\
\hline 28. & 6280.7 \\
\hline 29. & 6334. \\
\hline 30. & 6340.8 \\
\hline 31. & 6347. \\
\hline 32. & 6406. \\
\hline
\end{tabular}

\begin{tabular}{|c|c|}
\hline 33. & 6453.5 \\
\hline 34. & 6898.5 \\
\hline 35. & 6905.7 \\
\hline 36. & 7027.7 \\
\hline 37. & 7081.1 \\
\hline 38. & 7110. \\
\hline 39. & 7411.1 \\
\hline 40. & 7745.4 \\
\hline 41. & 7875. \\
\hline 42. & 8310.6 \\
\hline 43. & 8317.2 \\
\hline 44. & 8349.3 \\
\hline 45. & 8354.7 \\
\hline 46. & 8917.9 \\
\hline 47. & 8928.4 \\
\hline 48. & 9071.9 \\
\hline 49. & 9440.5 \\
\hline 50. & 9500.5 \\
\hline
\end{tabular}

TABLE 11

Model (A4) $>$ Modal (A5) $>$ Solution (A6) $>$ Solution Information

\begin{tabular}{|r|c|}
\hline Object Name & $\begin{array}{c}\text { Solution } \\
\text { Information }\end{array}$ \\
\hline State & Solved \\
\hline Solution Information \\
\hline Sewtion Output & Solver Output \\
\hline Identify Element Violations & 0 \\
\hline Update Interval & $2.5 \mathrm{~s}$ \\
\hline Display Points & All \\
\hline FE Connection Visibility \\
\hline Activate Visibility & Yes \\
\hline Display & All FE Connectors \\
\hline Traw Connections Attached & All Nodes \\
\hline Line Color & Connection Type \\
\hline Visible on Results & No \\
\hline Line Thickness & Single \\
\hline Display Type & Lines \\
\hline
\end{tabular}


"Mircea cel Batran" Naval Academy Scientific Bulletin, Volume XX - 2017 - Issue 1

The journal is indexed in: PROQUEST / DOAJ / Crossref / EBSCOhost / INDEX COPERNICUS / DRJI / OAJI I JOURNAL INDEX / I2OR / SCIENCE LIBRARY INDEX / Google Scholar / Academic Keys/ ROAD Open Access I Academic Resources / Scientific Indexing Services / SCIPIO / JIFACTOR

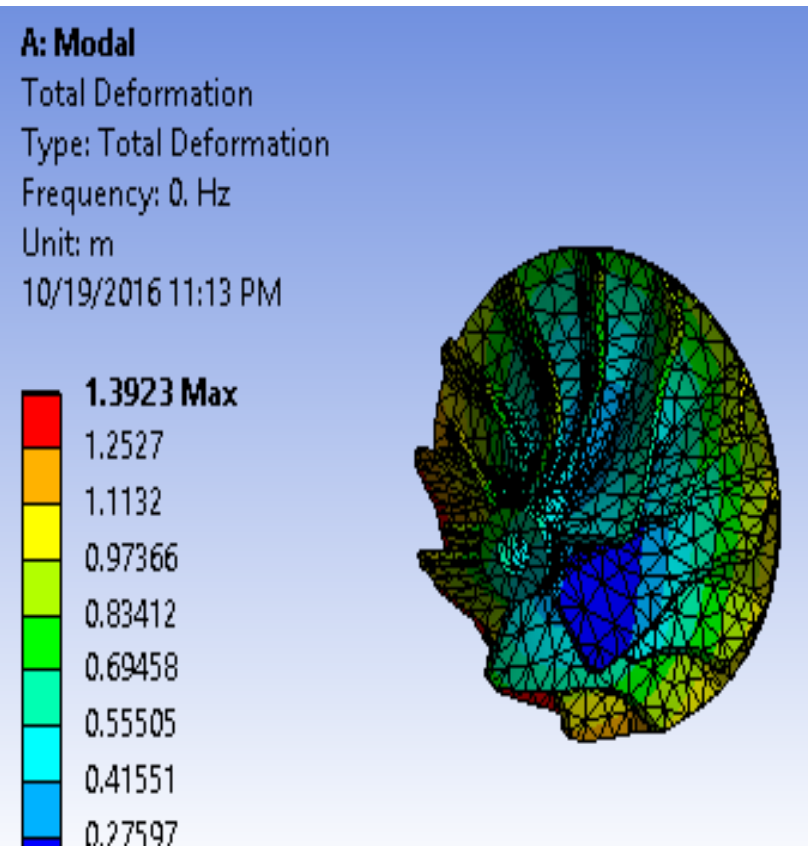

Figure 4. Total deformation on 3D turbine model

A: Modal
Total Deformation 2
Type: Total Deformation
Frequency: 0. Hz
Unit: m
10/19/2016 11:14 PM
\begin{tabular}{|l|l}
1.0893 Max \\
1.0074 \\
0.92543 \\
0.84349 \\
0.76155 \\
0.67961 \\
0.59767 \\
0.51574 \\
0.4338
\end{tabular}

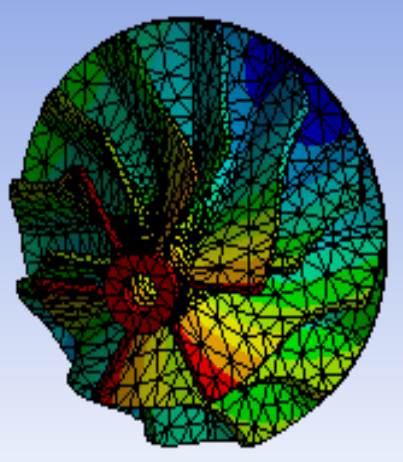

Figure 5. Total deformation on 3D turbine model

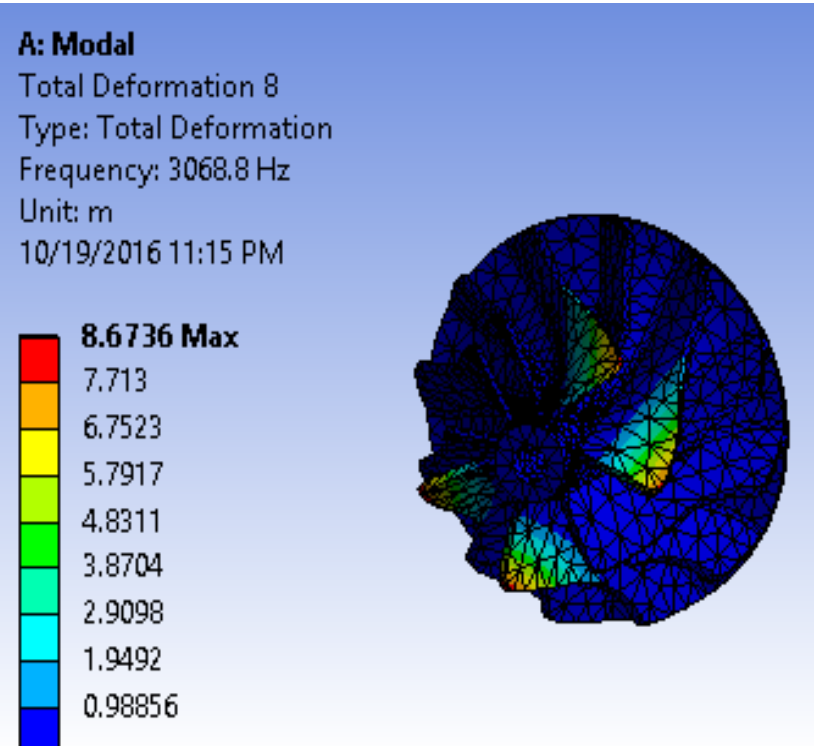

Figure 6. Total deformation on 3D turbine model

A: Modal
Total Deformation 10
Type: Total Deformation
Frequency: $3102.4 \mathrm{~Hz}$
Unit: m
10/19/2016 11:15 PM
\begin{tabular}{ll}
\hline 8.6987 Max \\
7.7323 \\
6.7659 \\
5.7995 \\
4.8331 \\
3.8667 \\
2.9003 \\
1.9339 \\
0.9675
\end{tabular}

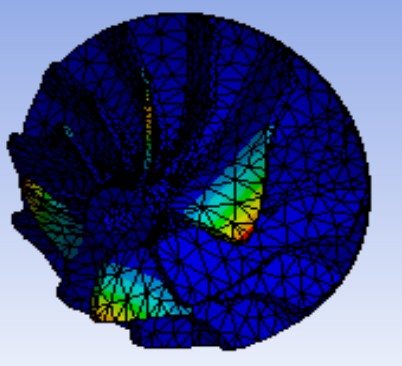

Figure 7. Total deformation on 3D turbine model 
"Mircea cel Batran" Naval Academy Scientific Bulletin, Volume XX - 2017 - Issue 1

The journal is indexed in: PROQUEST / DOAJ / Crossref / EBSCOhost / INDEX COPERNICUS / DRJI / OAJI I JOURNAL INDEX I I2OR / SCIENCE LIBRARY INDEX / Google Scholar / Academic Keys/ ROAD Open Access I Academic Resources / Scientific Indexing Services / SCIPIO / JIFACTOR

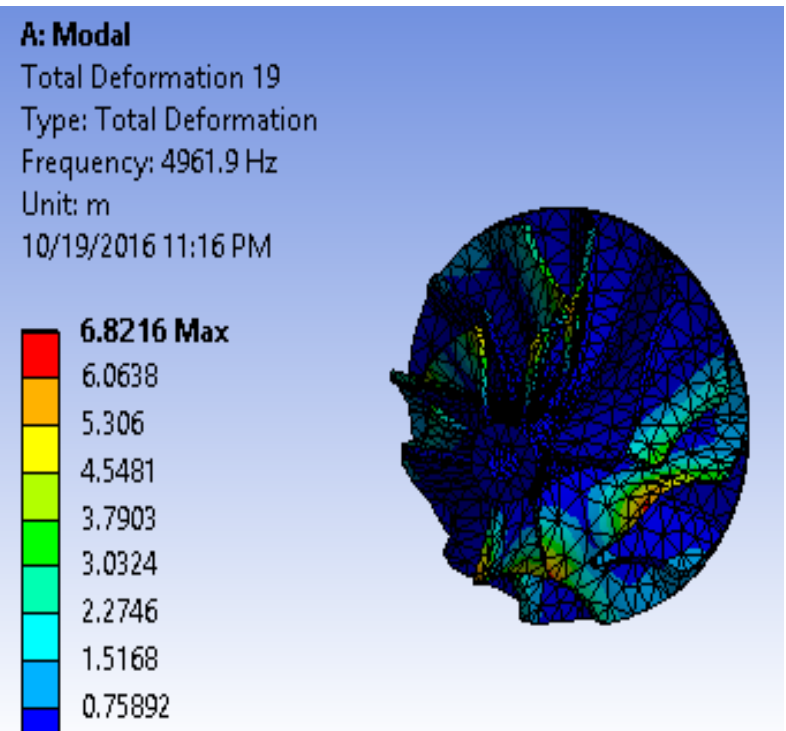

Figure 8. Total deformation on 3D turbine model

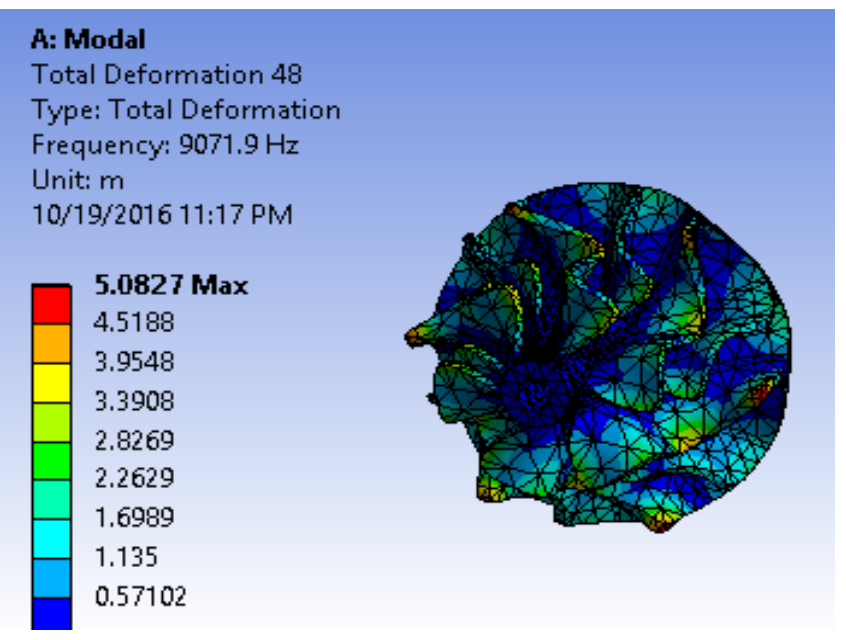

Figure 9. Total deformation on 3D turbine model

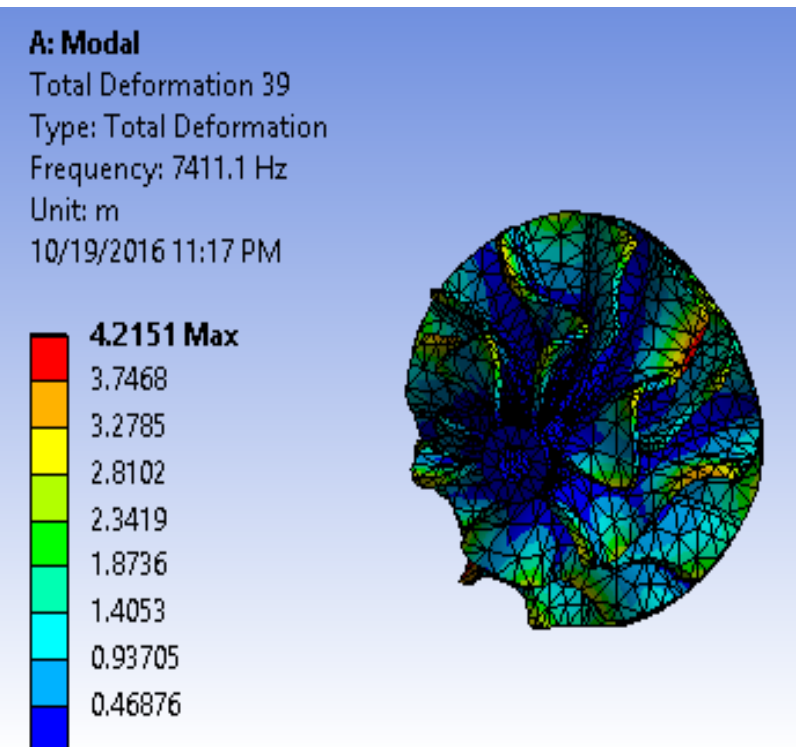

Figure 10.Total deformation on 3D turbine model

\section{Conclusion}

In this study, a modal method was used for an actual case study simulation based on turbine model. Due to high difficulties in making experimental tests on the presented turbine model, the Ansys method of numerical simulation is preferable. The proposed Ansys simulation made on turbine model is sufficiently precise in modal results. All results were presented as bearing deformation for a more visible effect of determined frequency. The Ansys simulation presented for turbine is a fast way to solve modal analysis for any bearing and it can be done using only a simple CPU unit with few resources. Frequencies obtained in simulation will not interfere with normal operation of the turbine. All frequencies presented should be avoided inside naval equipment using turbine from any source.

\section{Bibliography}

[1] Martinas, G, Arsenie A., Lamba M., Numeric geometry optimization of an wed for duct pressure angle, length and radius, Constanta Maritime University Annals Year XV, Vol.22. 
"Mircea cel Batran" Naval Academy Scientific Bulletin, Volume XX - 2017 - Issue 1 The journal is indexed in: PROQUEST / DOAJ / Crossref / EBSCOhost / INDEX COPERNICUS / DRJI / OAJI I JOURNAL INDEX I I2OR / SCIENCE LIBRARY INDEX / Google Scholar / Academic Keys/ ROAD Open Access I Academic Resources / Scientific Indexing Services / SCIPIO / JIFACTOR

[2] Călimănescu I., Stan L. C., Computer fluid dynamics (CFD)study of a micro annular gear pump, Atom 2016, Conference Paper.

[3] N. Tandon and A. Choudhury, An analytical model for the prediction of the vibration response of rolling element bearings due to a localized defect, J. of Sound and Vibration, 205 (3) (1997) 275-292.

[4] Sergeev, S.V., Proshunin, D.V. \& Sergeev, Y.S. Russ. Calculation of errors in hole machining by ansys software, Engin. Res. (2012) 32: 515. doi:10.3103/S1068798X1205022X

[5] Wu T. Y. and Chung Y. L., Misalignment diagnosis of rotating machinery through vibration analysis via hybrid EEMD and EMD approach, Smart Materials and Structures, 18 (9) (2009) 095004.

[6] Kiral Z. and Karagulle $H$., Simulation and analysis of vibration signals generated by rolling element bearing with defects, Tribology International, 36 (2003) 667-678.

[7] CAD model available at www.grabcad.com. 